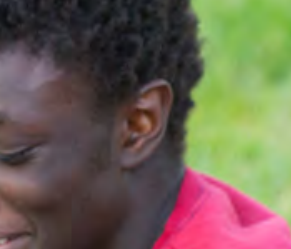

\title{
Is race linked to the structure of psychopathology in young people?
}

\author{
By Dr. Jessica Edwards
}

Researchers at the University of Wisconsin-Madison have found no significant difference in the hierarchical structure of psychopathology between African American and European American youths.

The rationale for Quanfa He and James J. Li's study came from two key issues in the field. First, that mental disorders tend to co-occur, ${ }^{1}$ suggesting that mental disorders may not be adequately described by our traditional diagnostic categories but instead may be better conceptualized in a hierarchical framework that transcends these diagnostic boundaries. ${ }^{2}$ Second, that there seem to be racial-ethnic differences in the prevalence of mental disorders in the USA. ${ }^{3} \mathrm{He}$ and Li thus aimed to clarify the structure of mental disorders across racial-ethnic lines.

The researchers studied $>8,000$ youths aged 8-21 from the Philadelphia Neurodevelopmental Cohort, who were interviewed to assess for 15 DSM diagnoses. They found that a hierarchical factor structure of mental disorders did not vary between African Americans (AA) and European Americans (EA) in this cohort, using multigroup confirmatory factor analyses. This suggests that mental disorders, or at least the way they covary in the population, are perhaps more similar between racial-ethnic subgroups than they are different.

The researchers note that additional studies are warranted to extend these findings to other populations. However, they believe that their finding reinforces the importance of prioritizing mental health research with diverse samples, to test whether any findings generated for one group generalize to at least one or more other subgroups. "Our demonstration of racial-ethnic 'invariance' might imply that some of our best evidencebased treatments can be effective for all, and not just for certain populations", proposes Li. "This hypothesis now needs to be tested".
Referring to:

He, Q. \& Li, J.J. (2020), Factorial invariance in hierarchical factor models of mental disorders in African American and European American youths. J. Child Psychol. Psychiatr. doi: 10.111/jcpp.13243.

References:

${ }^{1}$ Kessler, R.C. et al. (2005), Prevalence, severity, and comorbidity of 12-month DSMIV disorders in the National Comorbidity Survey Replication. Arch. Gen. Psychiatry. 62: 617-627. doi: 10.1001/ archpsyc. 62.6.617.

${ }^{2}$ Caspi, A. et al. (2014), The $p$ factor: one general psychopathology factor in the structure of psychiatric disorders? Clinical Psychological Science, 2, 119-137.

${ }^{3}$ Anderson, E.R. et al. (2010), Race/ethnicity and internalizing disorders in youth: A review. Clin. Psychol. Rev. 30: 338-348. doi: 10.1016/j.cpr.2009.12.008. 